\title{
Determination of Parachute Joint Factors using Seam and Joint Testing
}

\author{
Catherine Mollmann ${ }^{*}$ \\ Airborne Systems North America, Santa Ana, CA, 92704
}

This paper details the methodology for determining the joint factor for all parachute components. This method has been successfully implemented on the Capsule Parachute Assembly System (CPAS) for the NASA Orion crew module for use in determining the margin of safety for each component under peak loads. Also discussed are concepts behind the joint factor and what drives the loss of material strength at joints.

The joint factor is defined as a "loss in joint strength... relative to the basic material strength" that occurs when "textiles are connected to each other or to metals." CPAS engineering development phase, a conservative joint factor of 0.80 was assumed for each parachute component. In order to refine this factor and eliminate excess conservatism, a seam and joint testing program was implemented as part of the structural validation. This method split each of the parachute structural joints into discrete tensile tests designed to duplicate the loading of each joint. Breaking strength data collected from destructive pull testing was then used to calculate the joint factor in the form of an efficiency. Joint efficiency is the percentage of the base material strength that remains after degradation due to sewing or interaction with other components; it is used interchangeably with joint factor in this paper.

Parachute materials vary in type-mainly cord, tape, webbing, and cloth - which require different test fixtures and joint sample construction methods. This paper defines guidelines for designing and testing samples based on materials and test goals. Using the test methodology and analysis approach detailed in this paper, the minimum joint factor for each parachute component can be formulated. The joint factors can then be used to calculate the design factor and margin of safety for that component, a critical part of the design verification process.

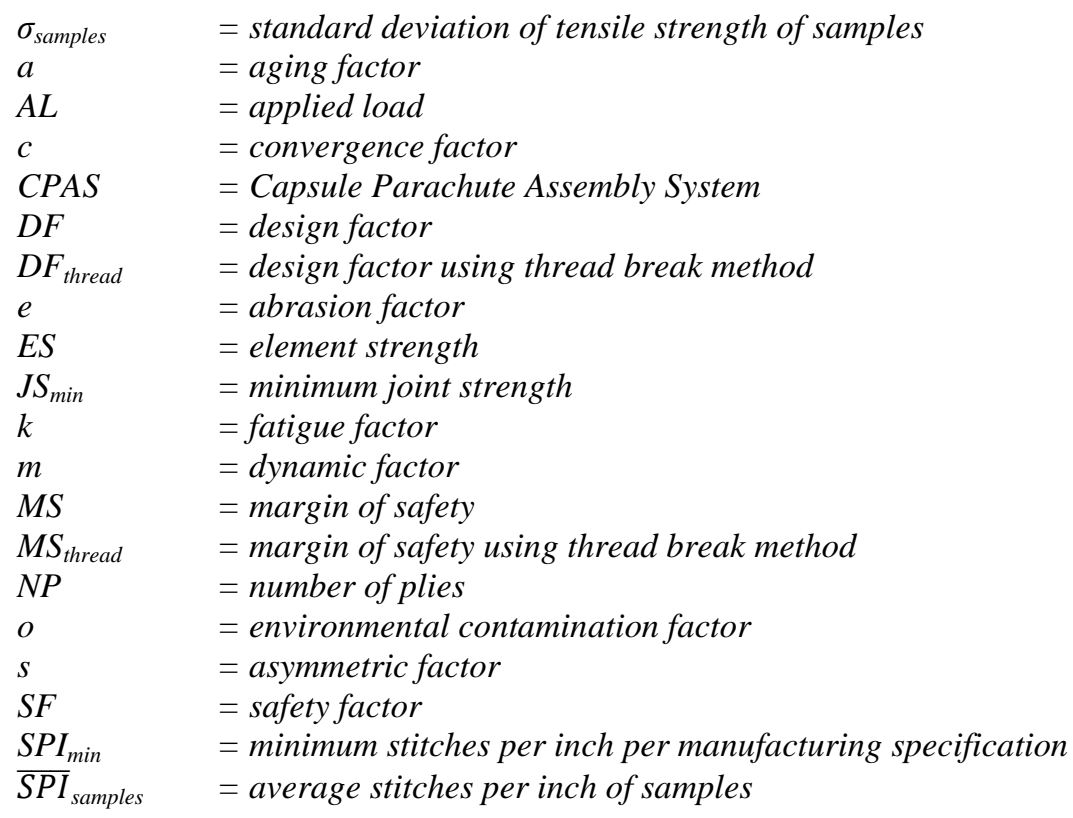

* Design Engineer, Space and Recovery Division, 3000 Segerstrom Ave, Santa Ana, CA, 92704, AIAA Member. 


$\begin{array}{ll}t & =\text { temperature factor } \\ u & =\text { joint factor } \\ \bar{x}_{\text {samples }} & =\text { average tensile strength of samples } \\ \bar{x}_{\text {thread }} & =\text { average tensile strength of thread }\end{array}$

\section{Introduction}

CPAS is the parachute recovery system for NASA's Orion Crew Module. The system deploys through four sequential phases-forward bay cover, drogue, pilot, and main-utilizing 11 parachutes total. CPAS has afforded an opportunity to study and optimize joint design on a large scale parachute development project.

An overview of the typical components of a ribbon parachute is shown in Fig. 1.

During parachute design, each component is designed in order to ensure adequate strength. This is measured by margin of safety, which is a measure of the excess strength of a component above the maximum applied load with degradation factors applied. When the margin of safety is positive, there is adequate margin. The higher the margin of safety, the more safeguard there is against any later increases to loads or degradation factors that often occur as development projects mature. When the system design requirements are finalized, any margins above zero indicate that excess strength (and weight) are present in the component design. A high margin of safety indicates that a material of lower strength, and thus lower weight, would be adequate to manage the same loads and degradations as the material of higher strength and weight. Optimizing the strength and weight relationship for all the subcomponents of a system is expensive, so materials comprising higher fractions of the total parachute mass, such as the suspension lines, are typically weight optimized whereas lesser components are designed primarily for structural adequacy.

Because textiles are constructed with a uniform composition throughout their length, parachute components are designed to be strong enough to have positive margin of safety at their weakest point. The weakest point of a component is generally where the joint efficiency, a measure of degradation due to sewing and joint formation, is the lowest. If the suspension lines are $50 \%$ of the weight of a parachute and one of the joints on the

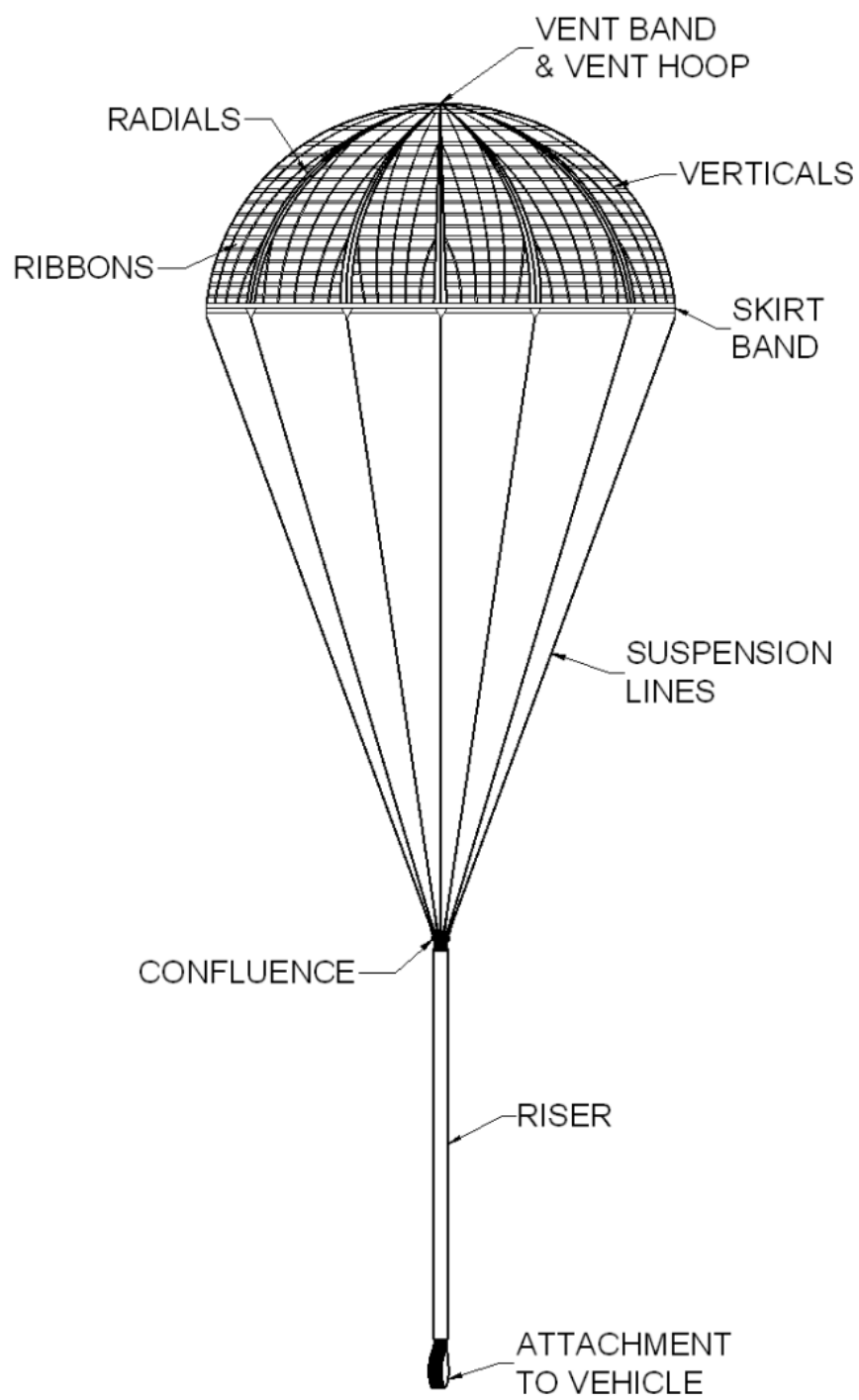

Figure 1. Overview of Parachute Components. suspension line has a joint efficiency of $80 \%$, then $(50 \%) *(100 \%-80 \%)=10 \%$ of the parachute mass is wasted because the excess used to compensate for the stress concentration is carried throughout the length of the component. Designing joints to achieve high efficiencies ensures that the weight penalty from joint degradation at one location is minimized. Weight optimization of parachutes requires efficient joint design in addition to optimum material selection. 


\section{Textile Strength and Degradation}

For parachute construction, textiles can be broken into three major categories: cord, tape/webbing, and cloth. For the purpose of this paper, tape and webbing are used interchangeably. All three categories consist of small elemental fibers that are either woven or braided together. Cord is typically braided. Tape and cloth are typically woven. In this paper, the term "warp fibers" will be used to describe the fibers of a textile that carry the majority of the load and are in the direction of the load axis. "Fill fibers" will be used to describe the fibers whose primary purpose is to organize the structure and facilitate load sharing.

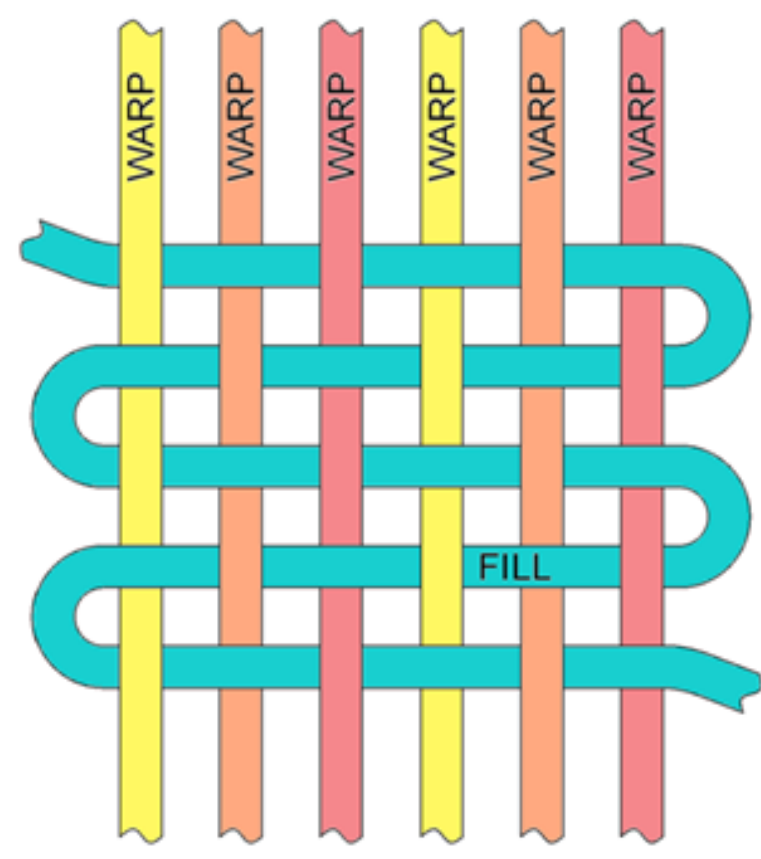

a)

Figure 2. Warp and Fill Fibers in Woven and Braided Textiles. The left image (a) shows the warp and fill fibers of a plain weave. The right image (b) shows the warp fibers of a braid.

Although tape and cloth consist of both warp and fill fibers, braided cord has only warp fibers. The first image in Fig. 2 shows the warp and fill fibers of a plain weave. The fill fiber interlaces with each warp fiber. The braided cord on the right of Fig. 2 is comprised of warp fibers only.

Because of differences in construction, braided cord tends to have a higher strength to weight ratio than woven tape or cloth. This is because the fill fibers in tape and cloth (up to half their mass) do not carry the applied load directly. All of the fibers in a braided cord carry some fraction of the applied load. For example, the CPAS main suspension line, $1800 \mathrm{lb}$ Kevlar ${ }^{\circledR}$ cord, has a $12 \%$ better strength to weight ratio than the radial, 1.00", $2400 \mathrm{lb}$ Kevlar® tape. Furthermore, the main suspension line is currently being replaced with a stronger and lighter braid that has a $45 \%$ better strength to weight ratio than the radial (and 30\% better than the current suspension line). Suspension lines and other long, unjointed structural members in parachutes are typically constructed from braided cord due to higher strength to weight ratio and the ease of constructing high-efficiency joints.

When a textile is sewn, the nominal woven path of the fibers is interrupted. The fibers near the stitches have a longer path length due to the extra distance required to pass around the stitches. These displaced fibers experience higher strain for an applied load than fibers in an uninterrupted part of the weave. Tension in the thread squeezes the yarns of the base material, which increases friction between the fibers to the detriment of load sharing. When the sample is loaded to failure, the displaced fibers reach their ultimate strength first, creating a localized failure that propagates through the sample. In Fig. 3a, an unsewn plain weave for tape or cloth is shown. The second image shows the same weave with a stitch through it - the adjacent fibers are displaced, creating a section of the material where the fibers have a longer path length relative to the unaffected section. The fibers near the stitch are likely to fail first when the sample is loaded to its ultimate strength.

Sewing through woven or braided textiles interrupts the weave or braid, which reduces load sharing between warp fibers. Although cord typically has a higher strength to weight ratio compared to tape and cloth, braided cord responds more poorly to sewing. The fill fibers in webbing and tape serve to transfer some load from more highly loaded to less highly loaded warp fibers. Woven textiles with dense fill fibers are capable of equalizing the warp fiber loading within a short distance of the weave interruption. Cord, due to lack of fill fibers, is less efficienct at load sharing than webbing. For this reason, the effect of stitching is more damaging to cord than webbing, although some equalization of load between yarns in cord is accomplished through friction within the braid. CPAS experience has shown lower efficiencies for joints where members are joined to suspension lines by sewing and higher efficiencies where members were sewn to webbing, even given denser stitch patterns in the webbing joints. 

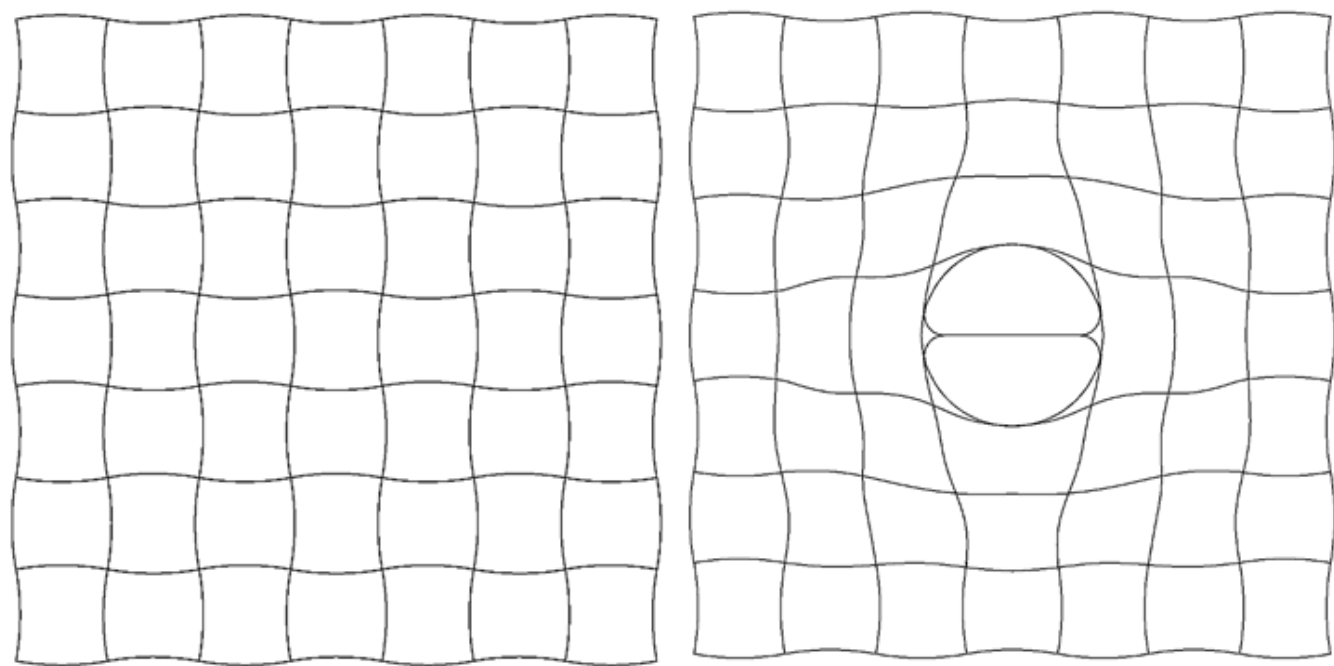

a)

b)

Figure 3. Fibers in a Plain Weave. The left image (a) shows an uninterrupted plain weave. The right image (b) shows the weave interrupted by a stitch.

In textiles with finer weaves, each warp fiber travels a longer path per unit length than in coarser weaves. This increased ratio of total warp fiber length to woven material length improves load sharing between warp fibers by reducing the relative stress concentration in the material due to weave interruptions from sewing. An optimized structural joint design may use webbing with more or less fill fiber content based on whether more benefit can be gained from higher strength to weight ratio (less fill material) or reduced joint loss due to sewing (more fill material.)

For cord, how tightly the material is braided affects the strength to weight ratio of the finished material. This is controlled by the material specification and is measured by the number of turns of the strands along the braid, known as the pick count. The first example in Fig. 4 shows a cord with 7 picks in a specified length; in the second example, there are 5 picks in the same distance. For the same weight per unit length, cord material with fewer picks per inch (Fig. 4b) will be stronger than material with more picks per inch (Fig. 4a). This is due to the angle of the fibers relative to the axis of the cord, referred to as helix angle. The greater this angle

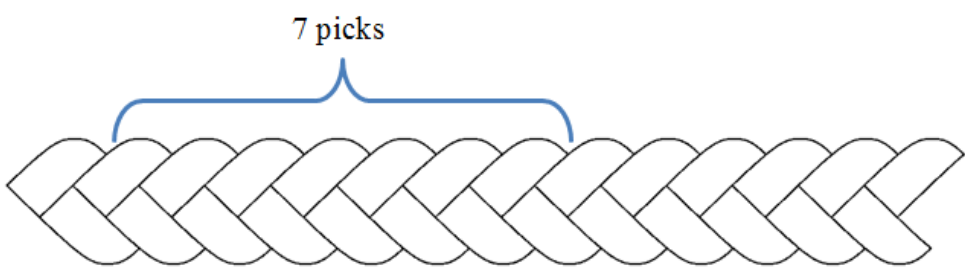

a)

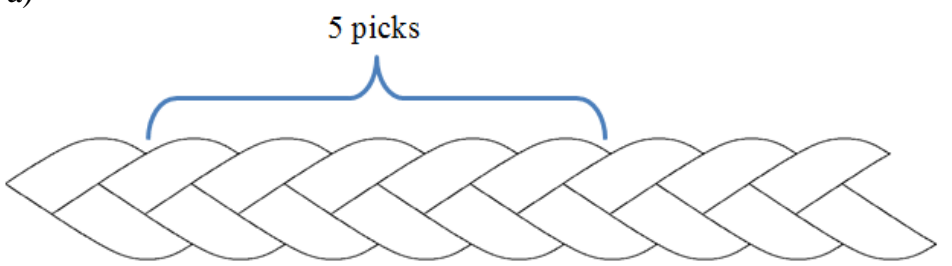

b)

Figure 4. Pick Count Measurements in Braids. The upper image (a) shows a denser braid than the bottom image (b). It has more picks per inch. (the greater picks per inch), the smaller the fraction of the fiber strength is used to carry axial load.

The legacy suspension lines on CPAS were replaced with new cord specifications that were stronger without increasing weight. This was done with a combination of reduced picks per inch (greater helix angle) and by removing excess fiber to bring the actual cord strength closer to specification strength. For example, on the CPAS forward bay cover parachute, the cord manufacturer was able to increase the specification strength of the suspension lines by $50 \%$--the number of picks per inch decreased from 8 to 6.5 and excess fiber was removed. Joint tests were repeated with the new cord specifications to ensure that suspension line joint efficiencies were not adversely affected by the change. Results showed that the joint efficiencies were similar, within $\pm 5 \%$ of the previous designs. 
Joint efficiencies were not harmed by reducing helix angle on CPAS because the insertions in the loop joints were already quite long so the design was tolerant of a small change to helix angle. A balance must be struck, as reduced helix angle makes the braid looser and more prone to snagging and also reduces the friction between the outer and inner cords of an insertion.

\section{Typical Parachute Joints}

Joints in tape, webbing, and cloth are typically sewn. The choice of the stitching pattern depends on the magnitude, direction, and type of load applied to the joint. Figures 5 and 6 below show two pieces of overlapping webbing sewn together:

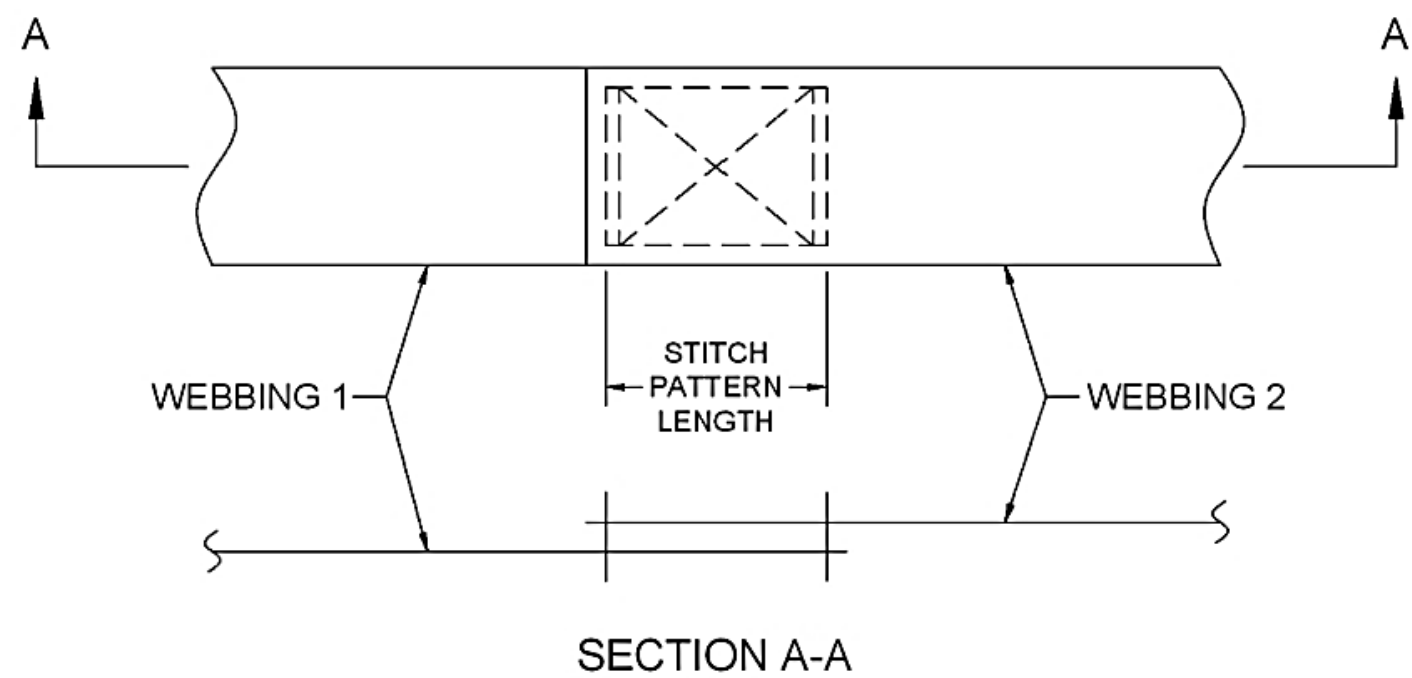

Figure 5. Box-x Stitch Pattern. Two pieces of webbing, as shown in Section A-A, are joined together with a box-x stitch.

B

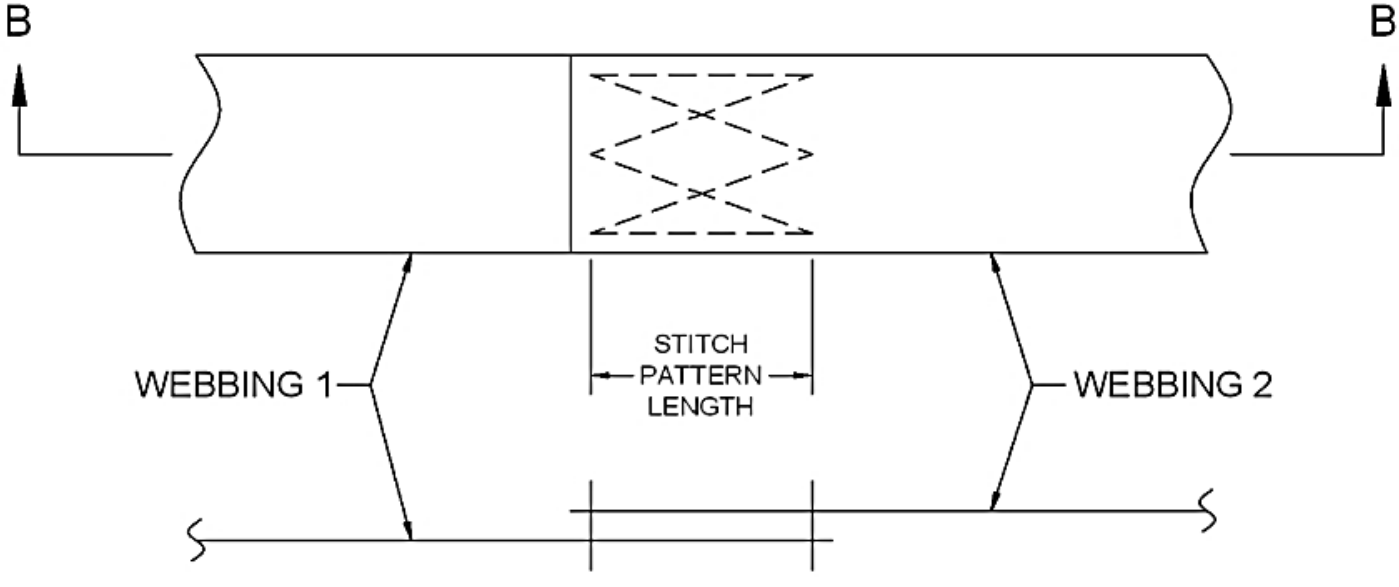

SECTION B-B

Figure 6. 3-Point Stitch Pattern. Two pieces of webbing, as shown in Section B-B, are joined together with a 3-point stitch.

The two stitch patterns are the same length but are implemented in different applications. The first example, a box-x stitch, is used when the stitch pattern could be loaded in various directions. There are stitches both parallel and perpendicular to the axis of the webbing, so a load either in the direction of the webbing or in an off-angle 
direction will be distributed efficiently without stress concentrations. Although not present in this example, a third piece of webbing attached at the box-x in the vertical direction could provide an off-angle load. Note the doubled rows of stitching at the left and right ends of the stitch pattern - these are used to ensure an even load distribution along the expected load axis.

The second example, a 3-point stitch, is used when the stitch pattern is engaged only along the axis of the webbing, since the majority of the stitches are sewn parallel to the two pieces of webbing. The transition of the stitch pattern from three stitches to six and back to three allows the load to transition and distribute efficiently. The 3-point stitch terminates with a single stitch at each corner, so the material (more significant in high modulus materials) will experience a stress concentration if pulled off-angle.

Other stitch patterns can be used to join two pieces of webbing or cloth, such as bartacks, 4- or 5-points, and parallel rows of straight stitching. All stitch patterns have advantages and disadvantages and must be chosen for each specific joint with attention. The strength of a stitch pattern, one important factor, can be estimated using Eq. (1):

$$
\text { Stitch Pattern Strength }=(\text { Length of Stitches })\left(S P I_{\text {min }}\right)(\text { Strength of Thread })(\text { De }- \text { rating factor })
$$

The length of stitches is measured only in the direction of load. The stitches per inch (SPI) is the minimum value permitted by manufacturing specification. The strength of the thread is the minimum value per thread specificiation. The de-rating factor, used to account for imperfect load-sharing and inefficiencies, is typically a value between 0.60 and 0.80 . This equation cannot be used to precisely predict the tensile strength of a joint; rather it is intended for a rough order of magnitude calculation for the purpose of prototype joint design prior to testing.

In order to attach cord to another component, a loop is often formed and then secured with an insertion. Figures 7 and 8 show typical insertions. The cord forms a loop and the tail is inserted back into the hollow center portion of the cord.

Cord insertions work by friction between the inner and outer layers. As the outer layer of cord is loaded, it compresses the inner layer of cord. This

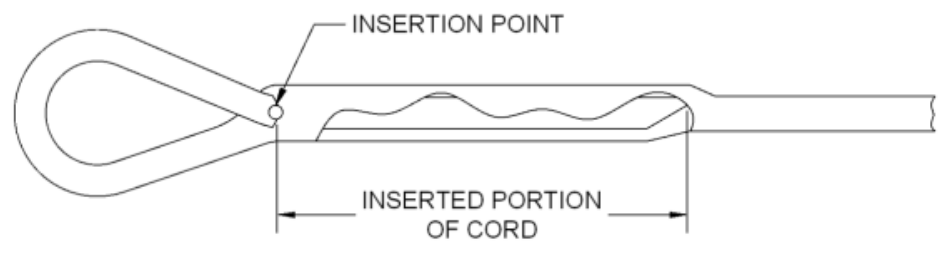

Figure 7. Cord Insertion. The inner cord is shown in the broken out section.

locks the two layers together so that the insertion cannot slip out. The length of the insertion depends on the cord size and material. The general rule used on CPAS is insertion lengths of 20-30 times

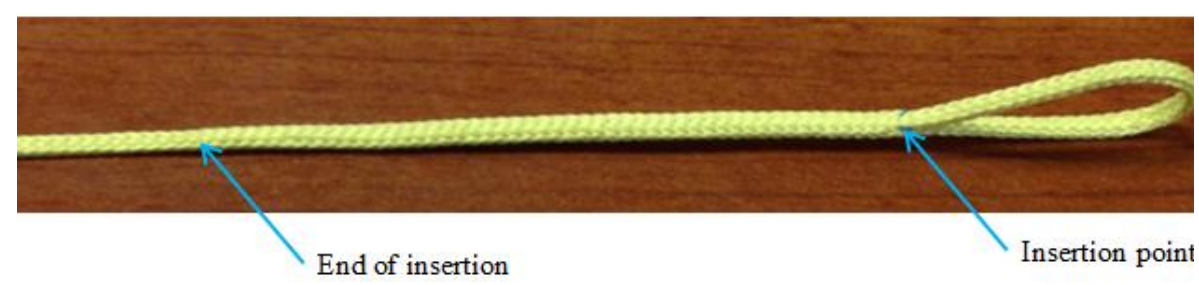

Figure 8. Insertion made with Small Kevlar ${ }^{\circledR}$ Cord the nominal diameter of the cord. For materials with lower coefficients of friction, such as Vectran ${ }^{\circledR}$ and Spectra ${ }^{\circledR}$, longer insertions are needed to prevent the inserted tail from pulling out of the cord. For materials such as Kevlar ${ }^{\circledR}$ and Nylon, which have higher coefficients of friction, shorter insertions can be used to produce the full cord strength without pulling out the inserted tail.

For any braided material, when the loop joint is initially loaded, the inserted tail can slip out a small amount before the outer cord is tensioned enough to lock it in place by friction. To prevent slipping, the insertion is sewn or tacked as shown in Fig. 9. This also prevents the insertion from slipping during handling and packing when no cord tension is available to provide friction. The strength of the stitch pattern or tack does not need to be

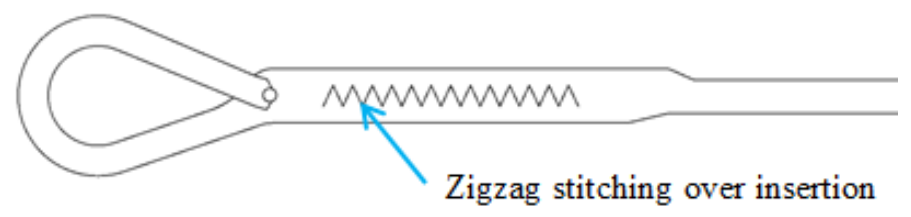

Figure 9. Sewn Insertion 
the full strength of the cord; it only needs to be strong enough to hold the insertion in place while the load is being applied and during handling.

\section{Parachute Loads and Degradation Factors}

The structure of each CPAS parachute is required to meet strict qualifications to ensure sufficient reliability for manned space flight. Each component of a parachute is designed to ensure adequate strength against predicted loads, which is expressed quantitatively through the margin of safety (MS) ${ }^{1}$, calculated below in Eq. (2):

$$
M S=\frac{E S * N P}{A L * D F}-1
$$

Where:

- The element strength (ES) is the minimum strength of the material per the specification.

- The number of plies (NP) is the quantity of layers of the material sharing the load.

- The applied load (AL) is a maximum value derived from analysis and testing.

- The design factor (DF) is a composite of all the degradation and de-rating factors.

The design factor (DF), for parachutes, is calculated by Eq. (3) ${ }^{1}$ :

$$
D F=S F * \frac{m * S * c}{u * e * k * o * t * a}
$$

The above factors, as defined in the nomenclature section, are explained below. The values used during initial CPAS engineering development are also given:

- SF, the safety factor, is used for all human-rated subsonic applications. For CPAS, 2.00 is used for safety critical components and 1.60 is used for all other components.

- $\mathrm{m}$, the dynamic factor, accounts for the effects of the dynamic loads at deployment. CPAS uses a dynamic factor of 1.05 for all textile elements.

- $\quad \mathrm{s}$, the asymmetric factor, accounts for uneven load distribution. CPAS uses an asymmetric factor of 1.10 for all textile elements.

- $\quad \mathrm{c}$, the convergence factor, is applied to suspension lines to describe the increased load due to the conical angle between suspension lines. CPAS uses a convergence factor of 1.05 for joints on the suspension line and 1.00 for all other locations.

- $\mathrm{u}$, the joint factor, measures the loss in strength of the base material due to joint formation. A conservative joint factor of 0.80 was initially applied on CPAS. The refinement and understanding of this value is the subject of this paper.

- e, the abrasion factor, accounts for element to element abrasion. CPAS uses an abrasion factor of 0.95, though some locations have been refined as a result of representative testing.

- $\mathrm{k}$, the fatigue factor, accounts for loss of material strength due to high pressure packing. CPAS uses a fatigue factor of 0.95 for packed components.

- $\mathrm{o}$, the environmental contamination factor, accounts for degradation due to sunlight, dirt, and contaminants such as ammonia and hydrazine. CPAS uses an environmental contamination factor of 0.94 for Kevlar ${ }^{\circledR}, 0.96$ for Nylon, and 0.91 for Vectran ${ }^{\circledR}$.

- $\mathrm{t}$, the temperature factor, accounts for degradation due to temperature exposure. CPAS uses a temperature factor of 0.95 for Kevlar ${ }^{\circledR}, 0.92$ for Nylon, and 0.86 for Vectran ${ }^{\circledR}$.

- a, the aging factor, accounts for long term storage of textile components. CPAS uses an aging factor of 0.96 for all textile elements.

For initial development, guess values for each degradation factor are often used, and CPAS started with values suggested by Knacke ${ }^{1}$. Analysis and representative testing allow the estimated degradation factors to be refined for the design of individual subcomponents. If the margin of safety is positive after application of the design factor and applied load, then the component has been adequately designed.

$\mathrm{u}$, the joint factor, is the subject of interest in this paper. Before joint factors had been tested, CPAS assumed 0.80 as a factor for all locations. This allowed for early estimates of design factor and margin of safety. This was considered conservative as most well-designed joints will not have joint factors this low; those that do can usually 
be redesigned to exceed 0.80 . In order to refine this 0.80 value during the next phase of parachute design, an empirical method called seam and joint testing was used which implements the actual materials and design of the parachute joint.

\section{Seam and Joint Testing}

Seam and joint testing evaluates degradation of base material due to the addition of or interaction with other components. In most cases, the joints being tested are sewn. Occasionally, components are joined with a looped connection or tied. Joints between textiles and the spacecraft structure (typically metal pins) are also tested using accurate interface geometry.

In order to isolate degradations due to joints, each tensile test sample is designed to test one joint interaction at a time. For example, a typical parachute radial would be split into five separate joints tests: radial at suspension line, radial at skirt band, radial at ribbons or panels, radial at vent band, and radial at vent hoop. For each joint test, a set of identical samples would be manufactured which duplicates the joint geometry and includes conditioned ends to be efficiently loaded into tensile test machine jaws. For each location where the sewing pattern or number of layers is changed over the length of an element, a new set of joint samples must be designed and tested.

Controls must be tested in order to accurately measure the degradation of base material strength due to interaction with other components. Controls are made of the same material as the component being tested and are tested to determine the manufactured strength of the material from which the samples are constructed. Textiles are constructed above their specified minimum tensile strengths, often by $25-40 \%$. Therefore, tensile tests of joints cannot be used to determine the strength lost due to joint formation without evaluating the as-manufactured strength of the individual lot of material used to construct the samples. Comparing test results to the minimum specified strengths would result in unconservatively high joint factors.

In order for the testing to be statistically relevant yet still succinct, each joint test on CPAS consists of ten samples and five controls. In order to compare the tensile strengths of the samples and controls, all 15 pieces must be constructed from the same lots of materials. A lot is a group of material that is constructed by the manufacturer from the same batch of raw material in a single production run. Otherwise, the differing strengths of materials might interfere with the joint factor determination. For CPAS tests, half of the ten joint samples are made by one set of operators and the other half are made by a different set of operators. Each group of operators uses different sewing machines. This ensures that any discrepancies in manufacturing method or operator ability will be visible in the test results

\section{A. Thread breaks}

It is usually considered preferable that the base material fail before the stitches holding the components together fail. This ensures that the full strength of the base material is being utilized, although too many stitches could disrupt the base material and lead to a low joint efficiency. During the joint tests where the stitches fail before the base material, the base material cannot be treated as the control material and the joint factor must be evaluated for the stitching failure mode.

During seam and joint testing on CPAS, a few sample sets had the majority of the samples fail in the stitching instead of the base material. For these cases, a different method was formulated for the determination of the margin of safety. The typical margin of safety calculation is adjusted from Eq. (2) to Eq. (4):

$$
M S_{\text {thread }}=\frac{J S_{\min }}{D F_{\text {thread }} A L}-1
$$

The element strength, ES, from Eq. (2) is replaced by the minimum joint strength, $\mathrm{JS}_{\min }$ :

$$
J S_{\text {min }}=\left(\bar{x}_{\text {samples }}-\sigma_{\text {samples }}\right)\left(\frac{E S_{\text {thread }}}{\bar{x}_{\text {thread }}}\right)\left(\frac{S P I_{\text {min }}}{\overline{S P I}_{\text {samples }}}\right)
$$

And the design factor, DF, from Eq. (2) is replaced by the thread design factor, $\mathrm{DF}_{\text {thread }}$ :

$$
D F_{\text {thread }}=S F * \frac{m * S * c}{e * k * 0 * t * a}
$$


Where:

- $\overline{\mathrm{x}}_{\text {samples }}$ is the average tensile strength of the samples with broken stitching.

- $\sigma_{\text {samples }}$ is the standard deviation of the tensile strengths of the samples with broken stitching.

- $\mathrm{ES}_{\text {thread }}$ is the element strength, or specification strength, of the thread.

- $\overline{\mathrm{X}}_{\text {thread }}$ is the average tensile strength of the thread used in the samples.

- $\mathrm{SPI}_{\min }$ is the minimum stitches per inch allowed per manufacturing specification.

- $\overline{\mathrm{SPI}}_{\text {samples }}$ is the average stitches per inch measured in the samples with broken stitching.

Note that Eq. (6) is identical to Eq. (3), except that the joint factor (u) is missing. This is because the joint factor is taken into account by Eq. (5).

Once the margin of safety is calculated for the joint which failed in the stitching, it is compared to the margin of safety calculated using the results from any tests where the actual base material failed. The lowest of these two results is used when reporting the margin of safety.

\section{B. Design of Samples and Controls}

Since the goal of seam and joint testing is to test the degradation of structural elements by joint formation, the material held by the test fixture must be the same as the base (control) material. Any sewing or interaction with other materials should end before the material meets the test fixture. The test section, where the joint is formed, is located in the middle of the sample, away from the jaws on either end. Although the stroke of the tensile testing machine must be considered, longer samples and controls are desirable in order to facilitate more load sharing and equalization among the fibers.

Each type of material requires different techniques to produce accurate and representative test results. Figure 10 below shows the standard set-up for constructing cord controls on CPAS. Loops are constructed at both ends to interface with the test fixture.
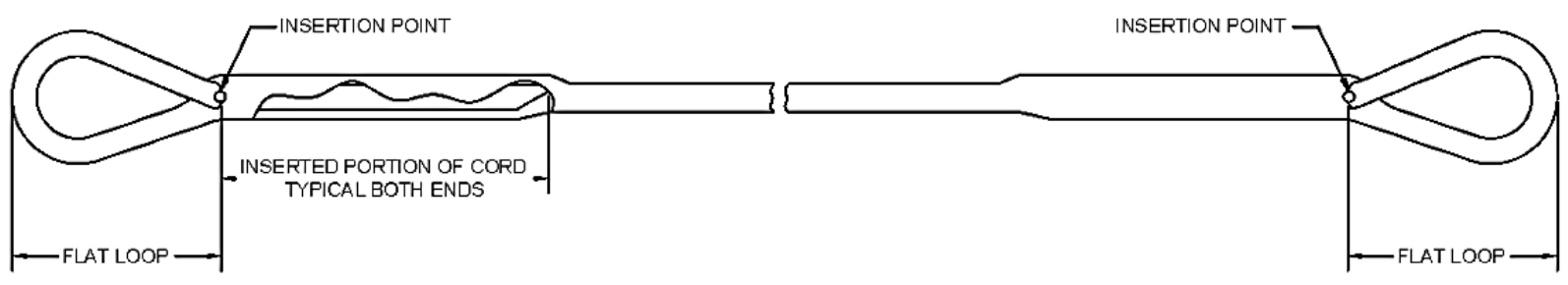

Figure 10. Typical CPAS Cord Control

When samples are made with cord as the base material, typically a gap is incorporated between the end of any joints or stitches and the beginning of the insertion used to create the test fixture-interacting loop. This isolates the effects of joint formation. See Fig. 11 below:

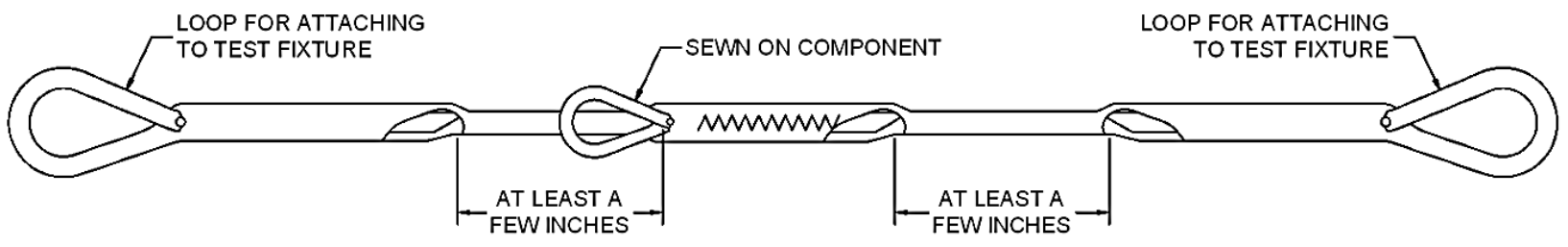

Figure 11. Typical CPAS Cord Sample. Note the gap on either side of the joint being tested. This is to isolate the test section from the insertions at either end used for attaching to the test fixture.

With the use of common test fixtures, as discussed in the next section, tape samples and controls can be loaded directly into the jaws without preparing the ends. A tape control should be long enough to mate with the fixtures and leave at least 12 inches between the test fixtures. Similarly, any samples constructed with tape should incorporate a section of plain material on either side of the sewn joint; this plain material will be loaded into the test fixtures. In Fig. 12 below, this section of material is shown on the left. The same gap of material, although not shown, is also on the right of the sewn on component. 


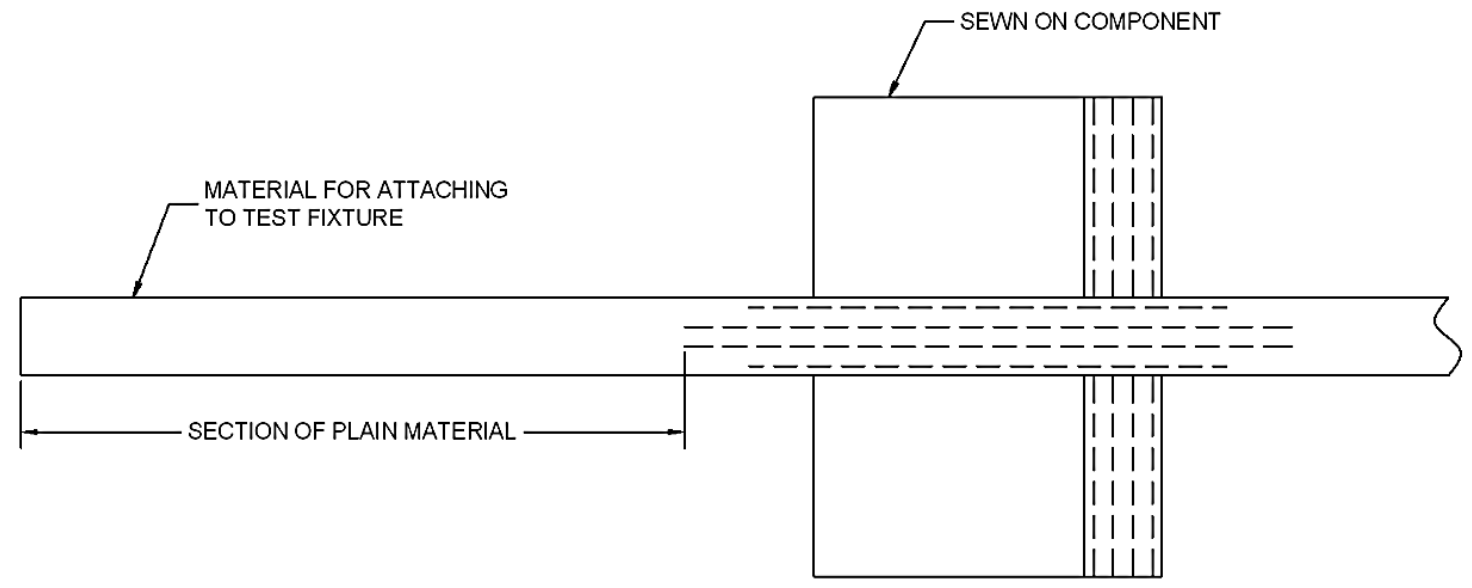

Figure 12. Typical CPAS Tape Sample. Note the length of base material on the left side of the joint. An identical length should be included on the right side.

The strength of cloth, such as the Nylon broadcloth used on the CPAS main parachute, varies based on cut width. Cloth samples must be carefully unraveled to specific widths to ensure the correct amount of fibers are loaded for accurate calculation of sample strength. The method used on CPAS was to order the cloth samples or controls to a certain width and then unravel the two long edges to a specified smaller width, as seen in Fig. 13. If the samples and controls are different widths, a correction factor has to be used to correct for the width difference.

\section{Test Fixtures}

During CPAS seam and joint testing, three machines were used to destructively test the samples. These machines included a 30k Tinius Olsen, which could break samples at loads of up to $30,000 \mathrm{lb}$. This machine was used for samples expected to break from 1,000 to $30,000 \mathrm{lb}$. If the samples were expected to break lower than 1,000 $\mathrm{lb}$, a Tinius Olsen machine with a $5,000 \mathrm{lb}$ capacity was used. The third machine, with a capacity of $150 \mathrm{lb}$, was used to test thread and small cord.

The 30k Tinius Olsen has two basic fixtures, one for cord and one for tape, with an additional fixture

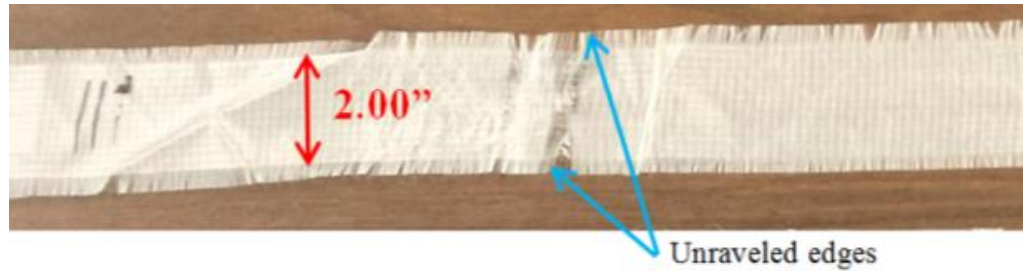

Figure 13. Typical CPAS Cloth Control. The control was scissor cut wider than the intended test section and then the edges of the control were unraveled to ensure 2.00 inches of continuous fibers.

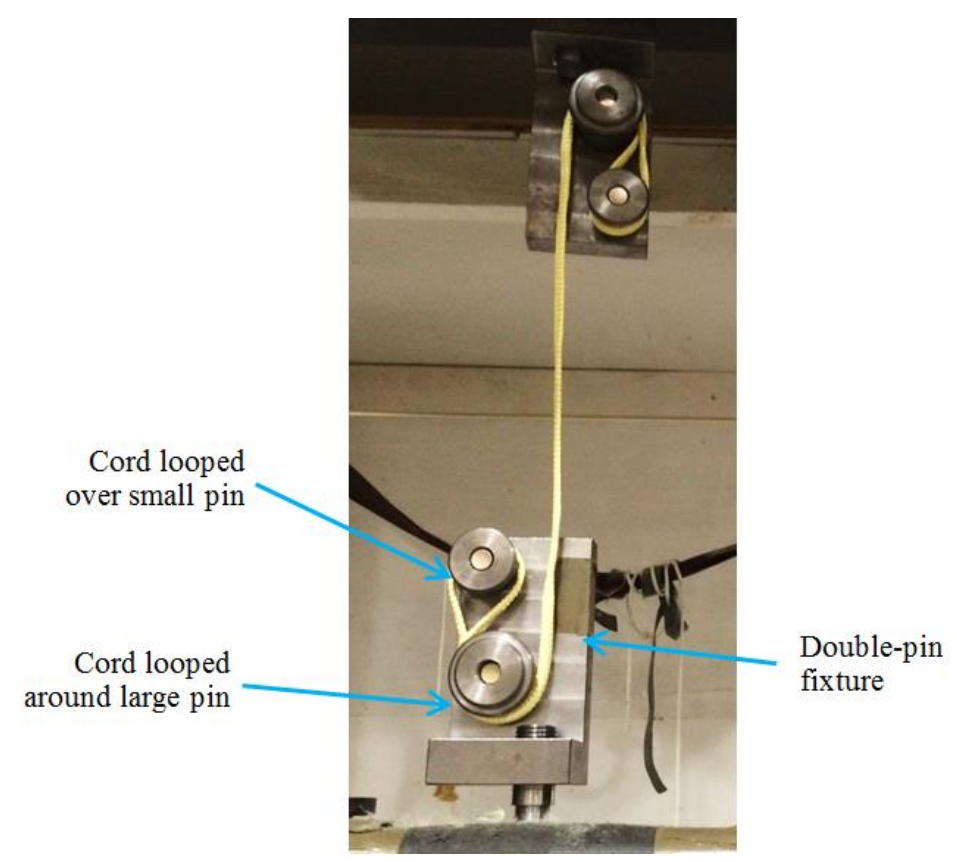

Figure 14. CPAS Cord Sample Installed in 30k Tinius Olsen with Double-Pin Grips

American Institute of Aeronautics and Astronautics 
custom designed to test materials passed over pins. The test fixture for cord consists of a double-pin arrangement where the cord wraps around the larger pin and then loops over the smaller pin as shown in Fig. 14. This has proven to be the method most likely to show the full strength of cord samples. Tensile strengths of cord measured by this method typically agree closely with the lot testing data from the cord manufacturer.

The test fixture for tape consists of Sedam grips, which use the tension in the sample to compress the grip around the material. Figure 15a shows an actual test set-up. Figure $15 \mathrm{~b}$ shows the routing of the material through the Sedam grips.

The 5k Tinius Olsen has a Sedam grip test fixture similar to the $30 \mathrm{k}$ Tinius Olsen which was used to test weaker tape and cloth using the same material loading configuration.

The third testing fixture, used to test pin efficiencies, is discussed in the next section.

One important consideration for joint testing is to ensure that the same fixtures are used for breaking both the controls and the samples. This guarantees as many similarities between the controls and the samples as possible and eliminates any potential differences from testing on different test fixtures. Also, correct test fixture choice is critical; using test fixtures that cause the material to break at the jaws can create artificially low tensile strength values. This leads to excess conservatism and a lack of understanding of the actual joint failure.

\section{Pin Efficiency Testing for Textiles}

A testing fixture was designed specially to meet the CPAS requirement to determine degradation factors due to small pin radii applied to strong materials. The pins used on the Orion crew module often have a design factor of only 1.60, which allows them to be light and narrow for the same maximum applied load as the textiles which carry design factors above 3.00. This meant accurate pin interface testing using circular pins was impossible because the strength of the textiles was so high that no pin could be built to carry the load at the prescribed diameter. A set of elongated pins was therefore designed to allow tensile tests of high strength textiles over small radii, the cross-section of which can be seen in Fig. 16.

Hard pin materials were chosen to prevent Kevlar ${ }^{\circledR}$ from printing the pin surface due to contact stress during high load. Figure 17 shows a pin secured in the pin efficiency
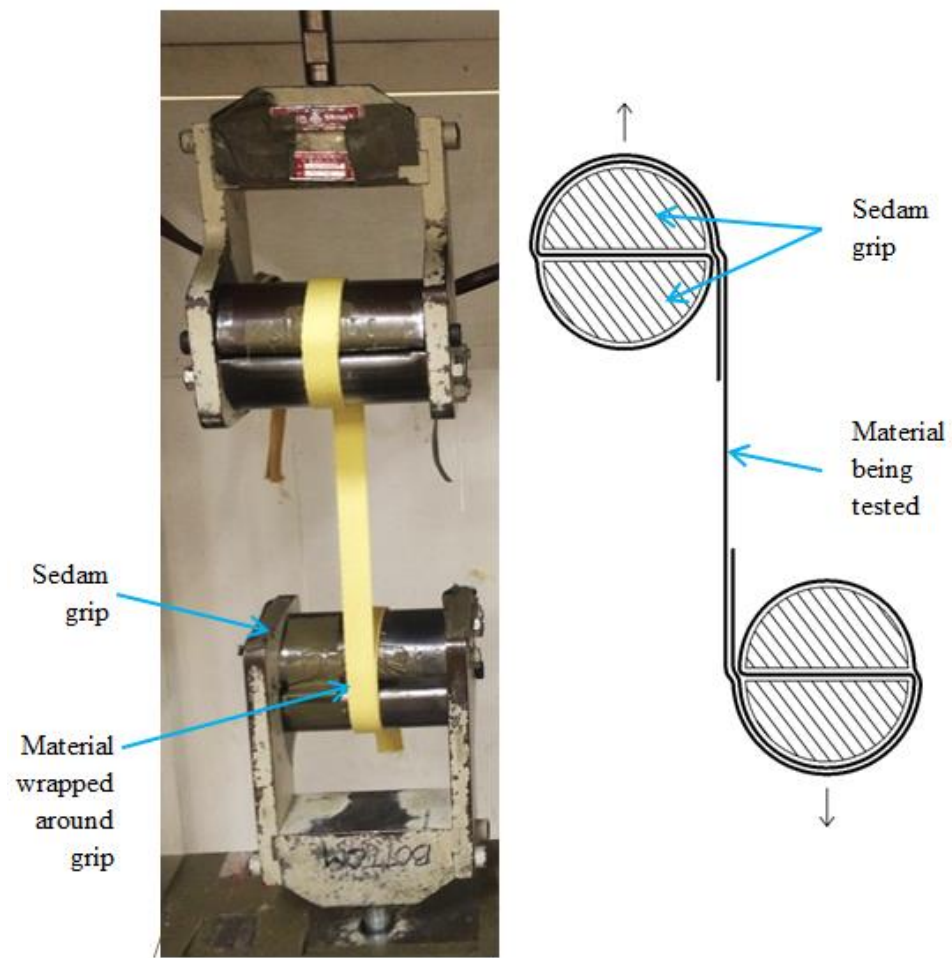

Figure 15. Typical CPAS Tape Sample and Cross-Section View. The left image (a) shows a typical tape set-up in the $30 \mathrm{k}$ Tinius Olsen with Sedam grips. The right image (b) shows the routing of the tape through the grips-as the grips move apart during the test, friction binds the material together. 
fixture. The two images shown in Fig. 18 show the front and side views of one of the custom pins.

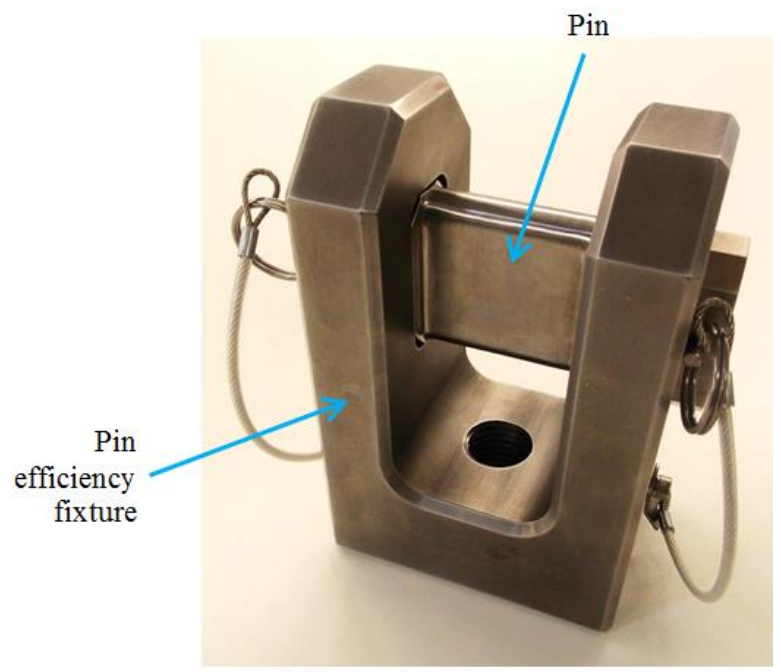

\section{Figure 17. Pin Efficiency Fixture with Pin Secured}

The pin efficiency fixture interfaces with one end of the 30k Tinius Olsen. Pins of various diameters could be secured into the fixture. The other end of the Tinius Olsen interfaces with the double-pin fixture (for cord) or the Sedam grips (for tape). For CPAS, a variety of pins were designed, including diameters ranging from 0.188 " to $0.70 "$, to match the geometry of the interfaces.

The following graphs, Figs. 20-22, show the joint efficiencies of three materials against pin diameter. All three of these materials comprise a component which interfaces with pins on CPAS. These types of charts can also be used during an initial design to balance pin size with required component strength.

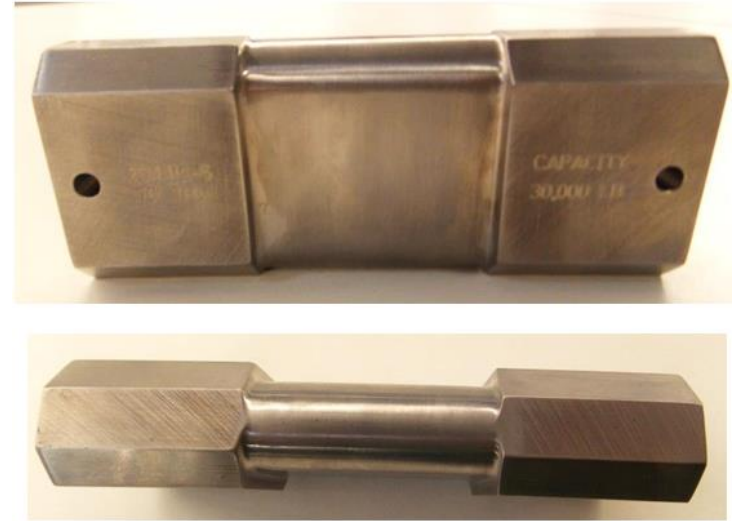

Figure 18. Typical Pin for Pin Efficiency Fixture

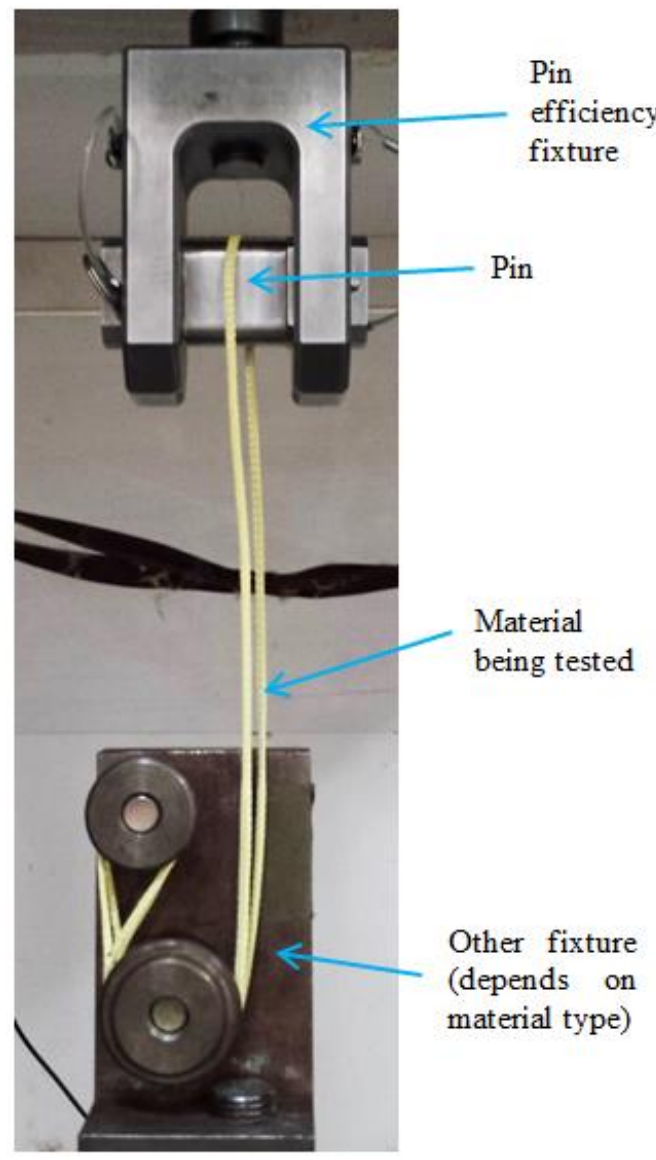

Figure 19. Pin Efficiency Fixture Installed in 30k Tinius Olsen. Since the material being tested is a cord, the double-pin fixture is used on the bottom end. 


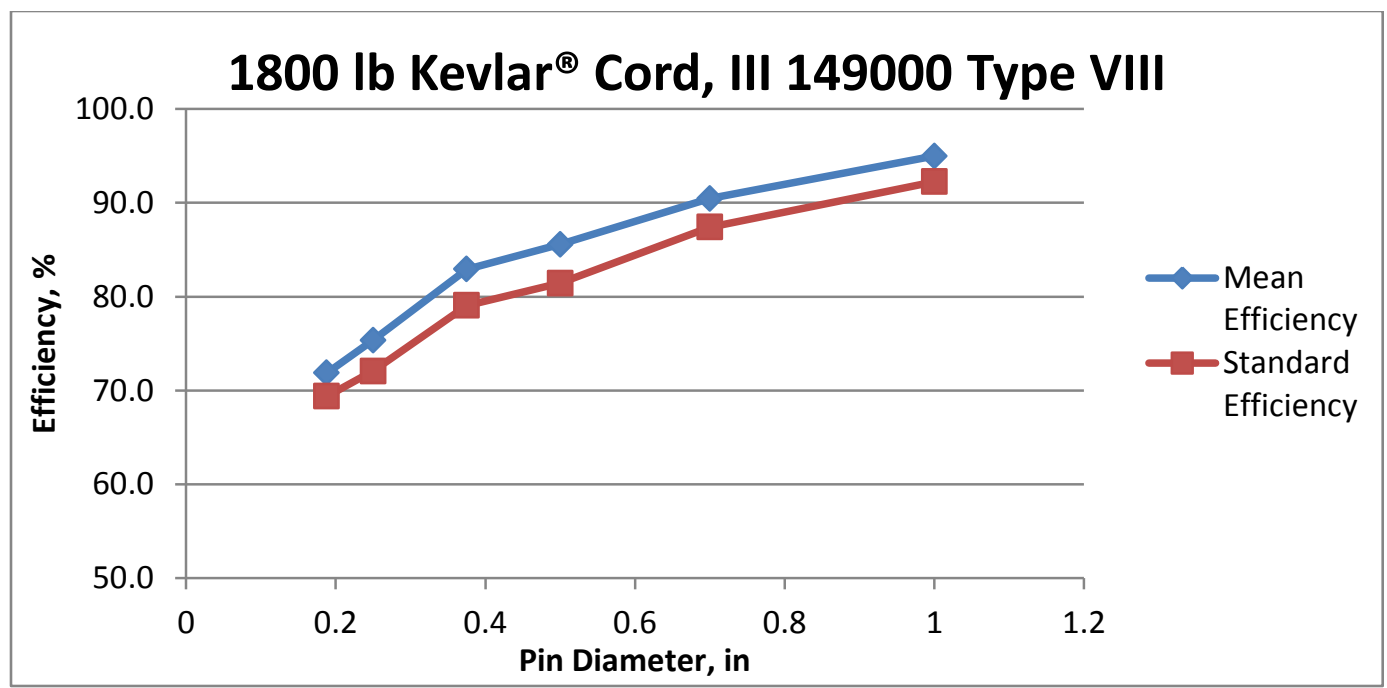

Figure 20. Joint Efficiency of $\mathbf{1 8 0 0}$ lb. Kevlar® Cord over Various Pin Diameters

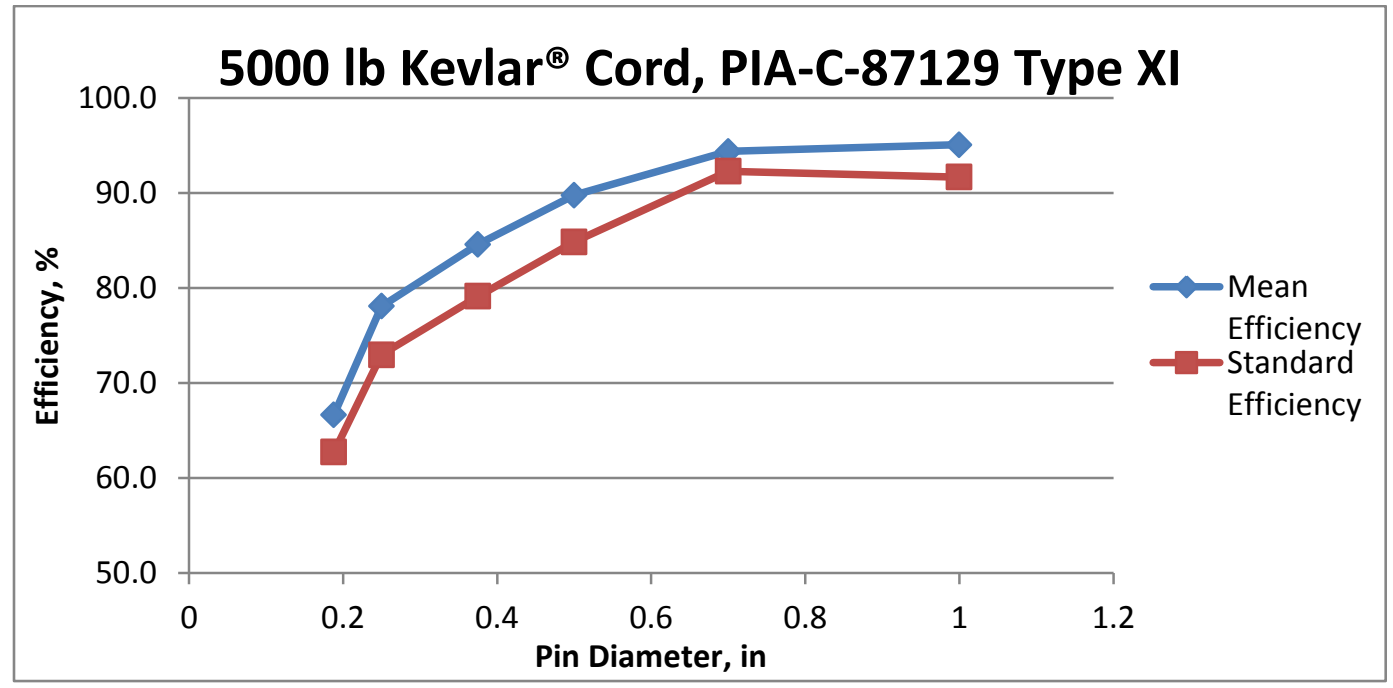

Figure 21. Joint Efficiencies of 5000 lb. Kevlar ${ }^{\circledR}$ Cord over Various Pin Diameters

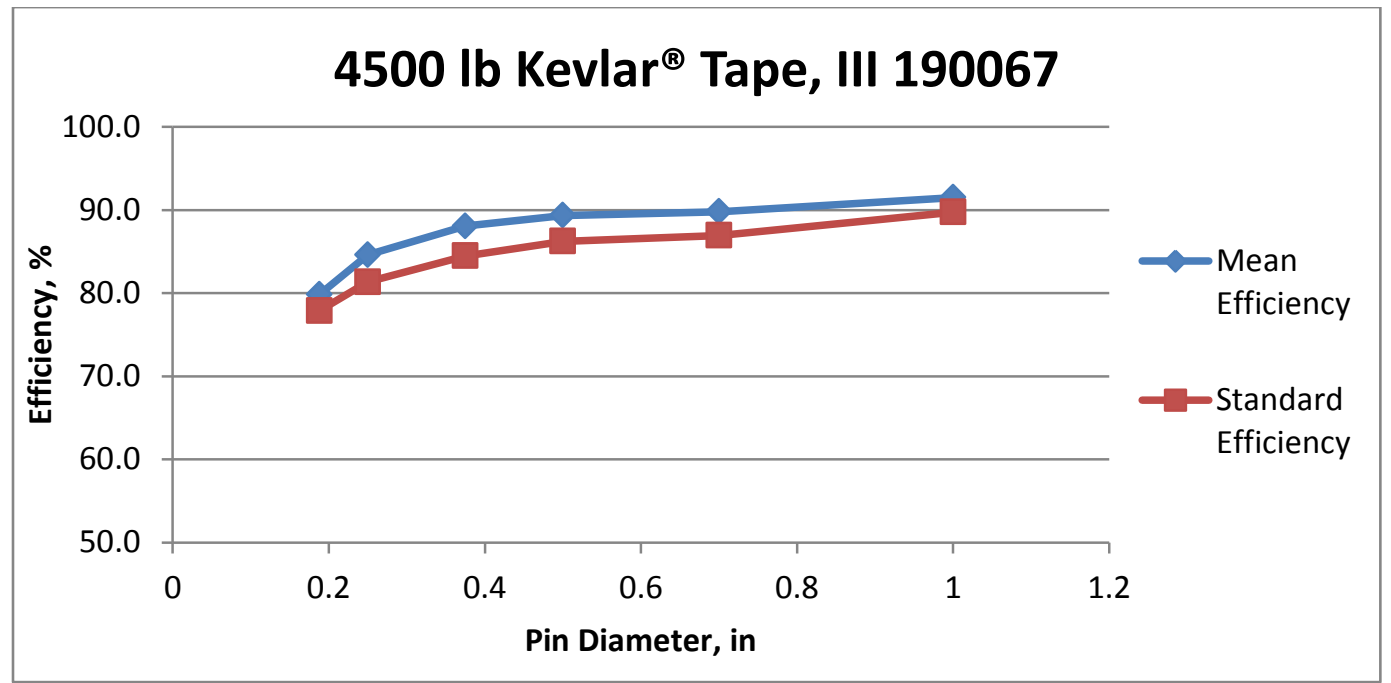

Figure 22. Joint Efficiencies of $\mathbf{4 5 0 0} \mathbf{l b}$. Kevlar® Tape over Various Pin Diameters 


\section{E. Running the Test}

Although the specific techniques for setting up and running a joint test vary according to the machine and operator, a few common guidelines apply. Samples and controls should be tested with the same test fixtures using the same machine program. For CPAS, a template was created for the $30 \mathrm{k}$ and $5 \mathrm{k}$ Tinius Olsen machines to ensure each test used the same machine speed and end conditions. This ensured uniformity among results from different sets of samples. On the 30k Tinius Olsen machine, the bottom test fixture moved downward at a constant speed of $12 \mathrm{in} / \mathrm{min}$. The test fixture stopped moving when the sample or control failed, which was defined as a decrease in load of $95 \%$ from the peak value. This peak value was then recorded as the tensile strength.

\section{F. Recording}

A test record sheet should be maintained for each joint test to record the data and test specific information. It can be instrumental in answering questions later when the family of joint tests is being analyzed. During CPAS seam and joint testing, the following sheet shown in Fig. 23 was used for each joint sample set:

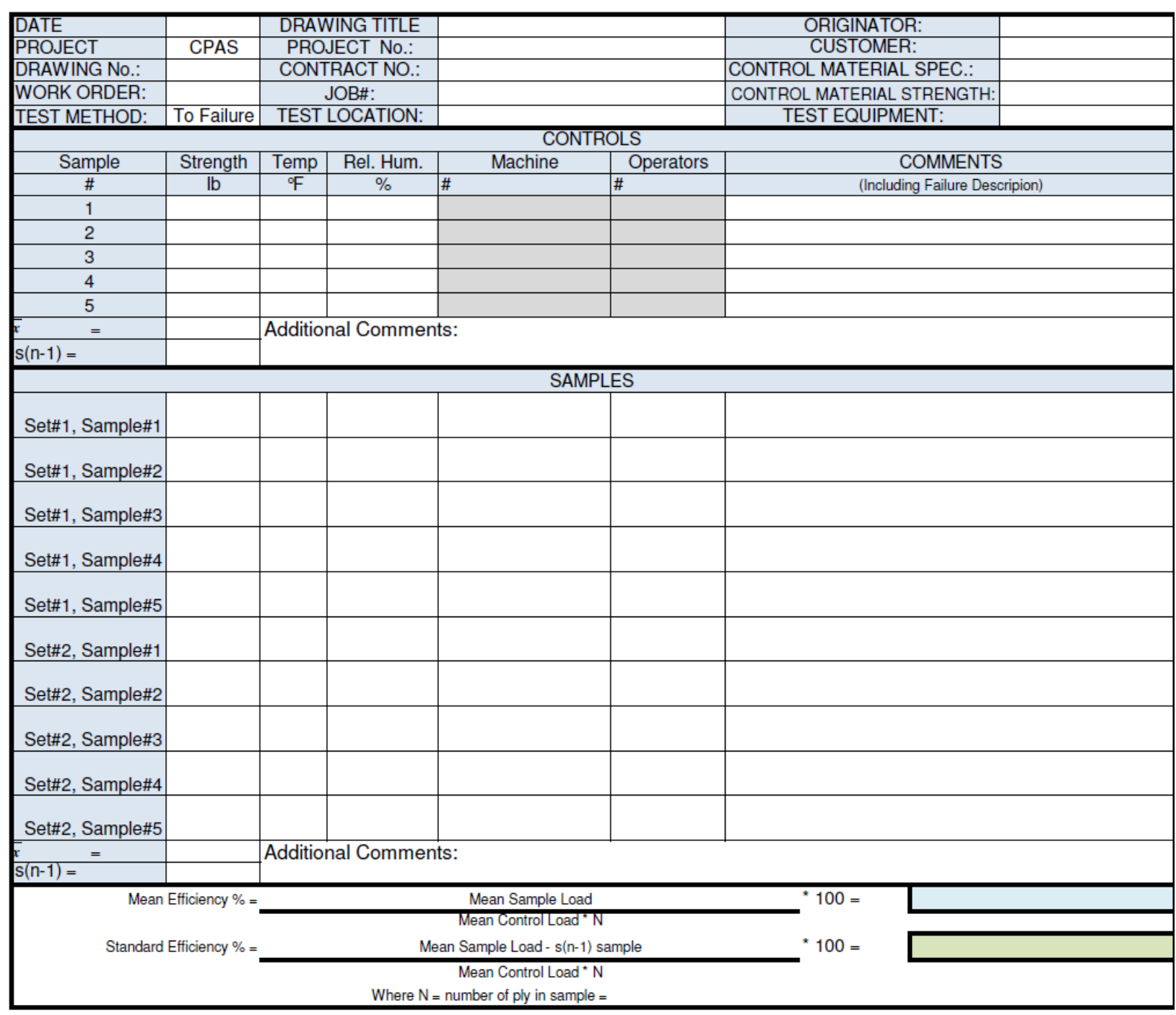

\section{Figure 23. CPAS Seam and Joint Test Record Sheet Template}

Any test record sheet should contain at least the following:

- Test set-up information: testing engineer, date, temperature, humidity

- Joint information: part number, description of joint, internal manufacturing identification

- Controls: specification type and strength, break force values and locations

- Samples: operator and machine identifications, break force values and locations

14

American Institute of Aeronautics and Astronautics 
Also, when the control and sample values are entered into the CPAS template shown in Fig. 23, the mean and standard efficiencies are calculated for the set of joint test results, as described in the next section.

In addition to a test record sheet, pictures should be taken of the samples and controls before and after testing. These pictures are used during later analysis of sample failure modes and are included in the test report. A comparison of the pictures and the tensile strengths can be used to analyze and explain any unusual results.

\section{G. Post-Test Analysis}

\section{Quantitative Analysis of the Joint Test Results}

Once the testing is complete, the sample and control tensile strengths can be used to calculate the joint factor, also called the joint efficiency. There are two different joint efficiencies that CPAS calculates. The first is the mean efficiency, calculated in Eq. (7):

$$
\text { Mean Ef ficiency }=\frac{\text { Average of sample breaks }}{(\text { Average of control breaks })(\text { Number of plies })}
$$

The second is the standard efficiency, calculated in Eq. (8):

$$
\text { Standard Efficiency }=\frac{\text { Average of sample breaks-standard deviation of sample breaks }}{(\text { Average of control breaks })(\text { Number of plies })}
$$

CPAS reports the standard efficiency as the joint factor $(\mathrm{u})$. The standard efficiency is more conservative than the mean efficiency, since it takes into account the standard deviation of the samples. Furthermore, it ensures that the majority of the test data support the margin of safety reported; if the mean efficiency was reported, the actual parachute joint could conceivably fail half of the time under maximum loading and degradation conditions with a margin of safety close to zero.

One of the factors in both of the above equations is the number of plies, which is the minimum number of layers of the base material at any location along the test sample. If there are two webbing layers that are continuous through the top and bottom test fixtures, the number of plies is two. For a test sample that is a single piece of cord with a loop at each end (as seen in Fig. 10 and 11), the number of plies would be one. If the test sample is one continuous loop of cord, then the number of plies is two. Effectively, for any type of material, the number of plies is the number of pathways that can transmit the load from one test fixture to the other. If the wrong value for the number of plies is used, the reported joint efficiency could be over $100 \%$ or under $50 \%$ and the error is easily identified.

\section{Qualitative Analysis of the Joint Test Results}

It is important that the testing be completed or observed by a cognizant engineer who is primarily responsible for ensuring correct test set-up and that the testing proceeds as expected. If the samples are breaking at unexpected locations or if the tensile strengths are unexpectedly high or low, it is important that the cognizant engineer is present to identify, document, and fix any identified problems.

When setting up, running, and analyzing the test, there are a few questions that should be asked:

- Is the design of the test samples identical to the joint on the actual parachute?

- Looking at the test set-up and sample construction, is the component being tested the one most likely to fail?

- Are samples breaking at the test fixture? Is this happening because the interaction between the sample and test fixture is artificially creating a weak spot or are the joints with other components not degrading the base material enough to cause it to break at those joints?

- Was the material that broke on each of the tests the same as what was identified as the control material?

- At least $60-70 \%$ of the samples should have the same component break.

- If the majority of samples failed in the stitches, apply thread break logic.

- Are there any outliers in the breaking values or locations of the samples or controls? Can these outliers be explained?

- Was the number of plies input correctly when calculating the joint efficiency?

These questions, coupled with an understanding of the joint and test objectives, should ensure a successful test. 


\section{Summary}

Parachute joints are constructed by means of sewing, looping, or tying. The formation of these joints degrades the strength of the base material, leading to a joint efficiency. This joint efficiency, or joint factor, is one of the many degradation factors used in the calculation of the design factor. The margin of safety, a measure excess strength of each parachute component, is calculated based on a comparison of the design factor and element strength with the maximum applied load. In order to optimize the weight of a parachute, each parachute component that constitutes a high fraction of the overall parachute mass must consist of joints with high joint efficiencies.

In order to measure the joint factor, each parachute component is split into discrete joint tests. The design of these tests should incorporate the use of carefully constructed representative joint samples and controls, appropriate tensile test machines and fixtures, accurate data recording at sufficient sampling frequency, and post-test analysis methods designed to reveal errors in testing. When joints fail in stitching, a different method must be used to calculated the margin of safety. A pin efficiency fixture can be used at locations where parachute components interact with a metal radius.

The methods described in this paper have been successfully implemented on CPAS during the engineering development phase in order to ensure adequate margins for all of the components in the four sequential parachute phases. With experience in testing and parachute design, adequately strong joints, and thus weight efficiency, can be pursued for all parachutes.

\section{Acknowledgments}

The author gives special thanks to Matt Zwicker, who provided invaluable advice and suggestions.

\section{References}

${ }^{1}$ Knacke, T. W., Parachute Recovery Systems Design Manual, $1^{\text {st }}$ ed., Para Publishing, Santa Barbara, California, 1992. 